\title{
Size Exclusion Coupled to Reversed Phase Liquid Chromatography for the Characterization of Cranberry Products
}

\author{
Inal Bakhytkyzy ${ }^{1}$, Oscar Nuñez ${ }^{1,2,3}$ and Javier Saurina ${ }^{1,2, \bigotimes}$ \\ ${ }^{1}$ Department of Chemical Engineering and Analytical Chemistry, University of \\ Barcelona. Martí i Franquès, 1-11, E-08028 Barcelona, Spain. \\ ${ }^{2}$ Research Institute in Food Nutrition and Food Safety, University of Barcelona, Recinte \\ Torribera, Av. Prat de la Riba 171, Edifici de Recerca (Gaudí), E-08921 Santa Coloma \\ de Gramenet, Barcelona, Spain. \\ ${ }^{3}$ Serra Húnter Fellow, Generalitat de Catalunya, Spain. \\ $\triangle$ Corresponding author: Javier Saurina \\ e-mail: xavi.saurina@ub.edu
}

\begin{abstract}
A combination of solvent extraction, size exclusion purification and reversed-phase liquid chromatography with UV absorption and fluorescence detection was here used for the determination of flavan-3-ols and related oligomeric compounds in cranberry products. The method relied on robust, simple and inexpensive instrumental equipment present in most of routine analytical laboratories. Sample extracts in methanol/water/hydrochloric acid solution $(60: 39: 1, v: v: v)$ were subjected to sizeexclusion chromatography (SEC) using Sephadex LH-20 as the sorbent to separate simple phenolic and oligomeric components. As a novel aspect of this work, the implementation of the SEC process in a reusable packed column was practically and economically highly advantageous. Fractions were analyzed by reversed-phase chromatography using a C18 core-shell analytical column and $0.1 \%$ formic acid aqueous solution and methanol as the components of the mobile phase. Chromatograms were recorded by UV absorption spectroscopy at $280 \mathrm{~nm}$ and by fluorescence spectroscopy at $\lambda_{\text {ex }} 280 \mathrm{~nm} / \lambda_{\mathrm{em}} 347 \mathrm{~nm}$. The method was applied to the determination of flavan-3-ols in various cranberry samples. Catechin, epicatechin and procyanidins A2 and B2 were found in different amounts depending on the cranberry varieties.
\end{abstract}


Keywords Cranberry; Flavanols; Procyanidin A2; Size-exclusion chromatography; Liquid chromatography

\section{Introduction}

Cranberries are a rich source of dietary flavonoids and phenolic acids of great interest for providing a variety of beneficial properties for our health, including antiproliferative, antioxidant, anti-inflammatory, antimicrobial and antineoplastic activities (He et al. 2006; Howell 2007; Pappas et al. 2009; Guay 2009; Coppo et al. 2014). In particular, flavan-3-ols, including catechin, epicatechin and their oligomeric derivatives (the so-called proanthocyanidins, PACs), have been recognized as one of the most significant families of polyphenols for health promotion.

PACs can be either singly linked through a bond from the $\mathrm{C} 4$ carbon on the upper unit to the $\mathrm{C} 6$ or $\mathrm{C} 8$ carbon of the lower unit (B-type), or doubly linked with a second interflavonoid bond formed between the $\mathrm{C} 2$ carbon of the upper unit and the $\mathrm{C} 7$ or C5 hydroxyl group of the lower unit (A-type). The presence of A-type linkages provides additional structural stability and rigidity to molecules. Besides, compounds rich in A-type links exhibit anti-adhesion activity which confers high antibacterial properties (Pappas et al. 2009; Guay 2009).

Methods to determine contents and structural features of PACs in cranberry products are required for quality control, characterization, and authentication purposes (Cote 2010). In this regard, the structural diversity of oligomeric PACs and the lack of commercially available standards are challenging analytical issues to be solved. PACs are often determined by HPLC methods (White et al. 2010; Navarro et al. 2014; Kraemer-Schafhalter et al. 1998; Lee 2013). It has been described that flavanol oligomers are hardly separated under reversed-phase mode, thus resulting in broad overlapping peaks (Diaz-Garcia et al. 2013). In contrast, the normal-phase mode working with silica stationary phases seems to be more recommendable for PAC separation leading to chromatograms with peak clusters that are eluted as a function of the degree of polymerization. Regarding detection, UV absorption and fluorescence (FLD) spectroscopies are often used. In particular, FLD offers enhanced selectivity and sensitivity since most of the interferences from other polyphenolic families are minimized from an appropriate selection of working wavelengths (Koerner et al. 2009, 
Bakhytkyzy et al. 2018). In addition, HPLC coupled to mass spectrometry (HPLC-MS) provides excellent analytical performance for the characterization and quantification of PACs (Koerner et al. 2009, Wang et al. 2012a). One of the main goals of MS is the possibility of discriminating among A- and B-type inter-flavan linkages. MS detection is often accomplished under positive ionization mode with electrospray (ESI) and atmospheric-pressure chemical ionization (APCI) sources to minimize the degradation of PAC molecules (Verardo et al. 2015; Lin et al. 2014; van Dooren et al. 2018). Apart from liquid chromatography, matrix-assisted laser desorption ionization (tandem) mass spectrometry (MALDI-MS/MS) is currently used for a more comprehensive structural elucidation of oligomeric PAC molecules (Monagas et al 2010). Recently, the topic of PAC structure elucidation based on MS has critically been reviewed by Rue and coworkers (Rue et al. 2018). Special emphasis has been paid in the most promising approaches based on LC-MS/MS and matrix-assisted MALDI. Other separation techniques such as gas chromatography (GC) and capillary electrophoresis (CE) have also been proposed for the determination of polyphenols such as flavanols in cranberrybased and other plant samples. GC is typically coupled to MS as a detection system (Wang et al. 2012b, Li et al. 2018). A precolumn derivatization using N,Obis(trimethylsilyl)trifluoroacetamide and trimethylchlorosilane reagents is recommended to decrease the relatively high polarity of analytes (Wang et al. 2011). In the case of CE, methods are often based on zone mode (CZE) with UV detection using fused-silica capillaries. Tetraborate buffer solutions ( $\mathrm{pH} 8$ - 10) with a small percentage of organic solvent (e.g., methanol or isopropanol) are used as running background electrolyte (Carrasco-Pancorbo et al. 2009). MS is combination with online preconcentration based on staking and related electromigration phenomena have been poposed in order to improve the sensitivity of the methods (Navarro et al. 2014, Parveen et al 2016, Sawalha et al. 2009).

Sephadex LH-20 is a hydroxypropylated dextran with proper cross-linking to yield a 3-D polysaccharide network specifically designed for the separation and purification of natural products (www.sigmaaldrich.com). Sephadex LH-20 swells differently in water and organic solvents. As a result, the wet particle size depends on the solvent affinity, varying from $1.5 \mathrm{~mL} \mathrm{~g}^{-1}$ for solvents such as toluene to $4.5 \mathrm{~mL} \mathrm{~g}^{-1}$ for dimethyl sulfoxide (DMSO) (Krenek et al. 2014). It is well known that swelling increases with solvent polarity, being maximum in DMSO, high in water and methanol, ca. $40 \%$ smaller in acetone and minimum in solvents of low polarity such as ethyl 
acetate or toluene. Sephadex particles strongly expand in polar solvents so only small molecules can pass through the gel bed (i.e., the sorbent is acting as a sieve to retain PAC molecules). Subsequently, when the column is treated with acetone or acetonitrile the size of beads shrinks allowing bigger molecules to elute. Sephadex gel is conceived as an ideal medium for separating naturally occurring phenolic compounds into nonpolymeric and polymeric fractions.

Size exclusion based on polymeric gels has been often applied to separate oligomeric phenolic compounds that occur in cranberry and other natural products. Apart from Sephadex, synthetic polymers based on hydroxylated methacrylic (Toyopearl) or polystyrene/divinylbenzene (PLgel) resins have been used as the sieving materials (Lerma-Herrera et al. 2017; Bindon et al. 2016; Gomez-Plaza et al. 2016; Duval et al. 2016). It has been found that Sephadex LH-20 displays high selectivity towards aromatic compounds in certain solvents so it has been used at analytical or industrial scale for the preparation of closely related species. For instance, Orejola et al. applied SEC with Sephadex to the fractionation of PAC oligomers of Ephedra sinica extracts of Chinese traditional medicine (Orejola et al. 2017). In another example, extracts isolated from Choerospondias axillaris were fractioned in five groups to be further characterized. Fractions differing in the degree of polymerization were analyzed by HPLC and antioxidant activities were assessed as well (Li et al. 2016). Different purification strategies were evaluated for the analysis of American cranberry products and the quantification of procyanidin A2. The study indicated that the procedure used highly influenced on the composition of the treated samples (Lee 2013).

In this paper, a new analytical method relying on size exclusion chromatography (SEC) using Sephadex LH-20 coupled to reversed-phase liquid chromatography (HPLC) with UV-absorption and fluorescence detection was developed to determine PAC compounds in cranberry. Sample extracts were subjected to SEC cleanup to separate the fraction rich in polymeric PACs from the monomeric constituents. Hence, simple polyphenols were first eluted from the gel using 30\% methanol. The solvent polarity was decreased using $70 \%$ acetone to accomplish the elution of the polymeric fraction from the corresponding increase of sieving size. Both fractions were analyzed by HPLC to compare their compositions and establish the performance of the cleanup step in fruits and pharmaceutical samples.

\section{Materials and Methods}




\section{Reagents}

Epicatechin and catechin (from Sigma-Aldrich), procyanidin A2 and procyanidin C1 (from PhytoLab, Vestenbergsgreuth, Germany) and procyanidin B2 (>98\% from Chengdu Biopurify Phytochemicals Ltd., Chengdu, China) were used to prepare $\sim 1000$ $\mathrm{mg} \mathrm{L}^{-1}$ stock standard solutions in methanol. Intermediate working solutions for method optimization and calibration were prepared by proper dilution in methanol/water $(1: 1$, $v: v)$ at $1,5,10,20,40,60,80$, and $100 \mathrm{mg} \mathrm{L}^{-1}$. All stock and standard solutions were stored in amber glass vials at $4^{\circ} \mathrm{C}$.

The mobile phase for the chromatographic separation was prepared with formic acid (Sigma-Aldrich, St Louis, MO, USA), methanol (UHPLC PAI-ACS SuperGradient, Panreac, Castellar de Valles, Barcelona, Spain) and Mili-Q Water purified using an Elix 3 coupled to a Milli-Q system (Bedford, USA). Methanol, acetone (UHPLC PAI-ACS SuperGradient, Panreac) and hydrochloric acid (37\%, Merck, Darmstadt, Germany) were used as the components of the extraction solutions. Sephadex LH-20 was from Sigma-Aldrich.

\section{Sample extraction}

Raw cranberry extracts, here referred to as E01 and E02, were kindly provided by Deiters S.L. (Barcelona, Spain) and corresponded to raw materials used for manufacturing dietary products and parapharmaceuticals. Nutraceuticals with preventive anti-cystitis activity were purchased from pharmacies in Gdansk (Poland) and Barcelona (Spain). These samples were coded as follows: C01 to C14 were commercial tablets and gelatin capsules containing cranberry extract amounts ranging from 175 to $425 \mathrm{mg}$; Sa01 and Sa02 consisted of sachets containing $240 \mathrm{mg}$ cranberry extract; Sy01 was a syrup with $12 \mathrm{mg} \mathrm{mL}^{-1}$ total PAC. Extracts were prepared by mixing $0.1 \mathrm{~g}$ of sample with $10 \mathrm{~mL}$ of methanol/water/hydrochloric acid 60:39:1 (v:v:v) solution. Samples were shaken in a vortex mixer (Genius 3 IKA, Staufen, Germany), sonicated for $30 \mathrm{~min}$ at room temperature (Branson 5510, Richmond VA) and centrifuged for $15 \mathrm{~min}$ at $3600 \mathrm{~g}$ (Rotanta $460 \mathrm{RS}$, Hettich, Tuttlingen, Germany). The resulting supernatant extracts were further treated by SEC as explained below. Sample extraction was carried out in triplicate to obtain independent extract solutions. 


\section{Size Exclusion Chromatography (SEC)}

Sample clean-up was performed by SEC with Sephadex LH-20. A XK 16/20 glass column (GE Healthcare Life Sciences, Uppsala, Sweden) was slowly packed with $1 \mathrm{~g}$ of Sephadex LH-20 sludge, preliminary swollen in 30\% methanol for $4 \mathrm{~h}$ at room temperature at $0.1 \mathrm{~mL} \mathrm{~min}{ }^{-1}$ flow rate using an Agilent 1100 system binary pump (G1312A). Prior to sample analysis, the column was equilibrated with 30\% methanol.

$1 \mathrm{~mL}$ of sample was loaded into the column using a manual injector and an Agilent 1100 system binary pump (G1312A) at $0.3 \mathrm{~mL} \mathrm{~min}^{-1}$. After loading, the column was washed with methanol/water 30/70 $(v: v)$ to remove the sugars and other small organic molecules. PACs were eluted with acetone/water 30/70 (v:v). The process was monitored by an Agilent 1100 system diode array detector (G1315B) at $280 \mathrm{~nm}$ and 520 $\mathrm{nm}$ to record the overall elution profile of polyphenols and colored compounds, respectively.

For off-line analysis, the acetone fraction was evaporated to dryness under $\mathrm{N}_{2}$ current and the residue was re-dissolved in $5 \mathrm{~mL}$ of mixed extraction solvent. The resulting solution was injected into the HPLC (see the following section). The on-line setup was based on introducing the SEC eluate to the HPLC injection port.

\section{Reversed-phase HPLC Method}

The chromatographic system consisted of an Agilent Series 1100 HPLC chromatograph (Agilent Technologies, Palo Alto, California, USA) with binary pump (G1312A), degasser (G1379A), automatic injection system (G1329B), diode array detector (G1315B) and fluorescence detector (G1321A). All these components were from the 1100 series except the automatic injector that belongs to the 1200 series. An Agilent ChemStation for LC (Rev. A. 10.02) software was used for instrument control and processing.

The chromatographic separation was carried out on a Kinetex C18 reversedphase (100 mm x $4.6 \mathrm{~mm}$ I.D., $2.6 \mu \mathrm{m}$ particle size) column with a Gemini C18 precolumn (4.0 mm x $6.0 \mathrm{~mm}$ I.D.) from Phenomenex (Torrance, California, USA). The elution program relied on $0.1 \%$ formic acid in water $(v: v)$ and methanol $(\mathrm{MeOH})$ as follows: 0 to $30 \mathrm{~min}$, $\mathrm{MeOH} 5 \rightarrow 35$ (v:v), linear increase); 30 to $40 \mathrm{~min}$, $\mathrm{MeOH} 35 \rightarrow 70$ $(v: v)$, linear increase). Subsequently, the column was cleaned for 2 min at $95 \% \mathrm{MeOH}$ and conditioned for $3 \mathrm{~min}$ at $5 \% \mathrm{MeOH}$. The flow rate was $0.4 \mathrm{~mL} \mathrm{~min}-1$ and the 
injection volume was $5 \mu \mathrm{L}$. Chromatograms were acquired at $280 \mathrm{~nm}$ by absorption, and at $\lambda_{\text {ex }} 280 \mathrm{~nm} / \lambda_{\text {em }} 347 \mathrm{~nm}$ by fluorescence detection (FLD) spectroscopy.

\section{Results and discussion}

\section{Extraction procedure}

Prior to fraction purification by size exclusion, the extraction of analytes with acetoneand methanol-based media was studied using $100 \mathrm{mg} \mathrm{L}^{-1}$ pure standard solutions of representative analytes including a dimmer (procyanidin A2) and two monomers (catechin and epicatechin). Apart from differences in the oligomeric nature, these compounds also differed in the polarity being catechin a model of polar flavonoids and A2 a model of less polar flavonoids (and epicatechin displayed an intermediate polarity). Methanol/water/HCl 60/39/1 (v:v:v), acetone/water/HCl 70/29.5/0.5 (v:v:v) and acetone/methanol/water/HCl 30/30/39/1 (v:v:v:v) extracting solutions were employed. This preliminary assay indicated that the performance of acetone and methanol solvents was similar.

Extraction was further studied with lyophilized cranberry samples using various acidified hydro-organic solutions. Samples (ca. $0.1 \mathrm{~g}$ ) were extracted by sonication for 30 min with $10 \mathrm{~mL}$ of solvent. It should be noted that the cranberry fruit extracts contained a wider range of molecules. As a result, information could be gained either regarding to individual and overall polyphenols. Results with methanol/water/HCl $60 / 39 / 1(v: v: v)$ and acetone/water/ $\mathrm{HCl} 70 / 29.5 / 0.5(v: v ; v)$ indicated that methanol seemed to be more recommendable. The influence of solvent percentage and $\mathrm{HCl}$ concentration on the recovery of selected analytes was assessed in a more comprehensive manner by experimental design. $\mathrm{MeOH}$ percentage was assayed at 3 levels $(0,40$ and $60 \% v: v)$ and $\mathrm{HCl}$ concentration at 4 levels $(0,0.5,1$ and $2 \%(v: v))$. Results shown in Fig. 1a indicated that A2 was better extracted with $60 \%$ methanol and $1 \% \mathrm{HCl}$ (catechin and $\mathrm{C} 1$-not shown here-, and epicatechin -Fig 1b-, displayed a similar behavior) while $\mathrm{B} 2$ recovery was maximum at $40 \%$ methanol and $2 \% \mathrm{HCl}$ (Fig. 1c). As a compromise for all these analytes, methanol/water/ $\mathrm{HCl} 60 / 39 / 1$ (v:v:v) was selected as the extraction solvent for the overall flavanol recovery (Fig. 1d).

\section{Size Exclusion Chromatography for the purification of cranberry extracts}


Extracts of cranberry products were fractioned by SEC to separate the PAC oligomers from other monomeric polyphenol components. As the sieving size of Sephadex decreased with solvent polarity, the procedure was started using an extraction solvent of high polarity (here, methanol/water/HCl 60/39/1 (v:v:v)) which allowed oligomeric PACs to be retained while small molecules went through the inter-particle sorbent bed. Once this fraction was eluted, the solvent was replaced by acetone/water 70:30 v:v to reduce significantly the size of Sephadex particles to accomplish the elution of bigger molecules including PACs.

Preliminary experiments were carried out in batch mode on a manual setup using homemade cartridges consisting of polypropylene empty cartridges $(50 \mathrm{~mL}$ capacity) filled with Sephadex LH-20 using a Supelco-57030-U Visiprep ${ }^{\mathrm{TM}}$ SPE Vacuum Manifold. After filling, sorbent devices were preconditioned overnight with methanol/water 30:60 $(v: v)$. The influence of sorbent amount on the purification process was assessed with $0.5,1,1.5$, and $2 \mathrm{~g}$ of Sephadex loaded in the cartridges. Bigger amounts could not be properly packed in the polypropylene containers. In the study, 10 $\mathrm{mL}$ of extracts were loaded in the cartridges at a constant flow rate of $1 \mathrm{~mL} \mathrm{~min}{ }^{-1}$, washed with $10 \mathrm{~mL}$ of methanol/water 30:70 v:v and eluted with $10 \mathrm{~mL}$ of acetone/water/HCl 70:29:1 (v:v:v). The performance of cleanup was estimated from the concentration ratio of procyanidin A2/epicatechin as a simply index of polymeric versus monomeric components. Best cleanup results were obtained with 0.5 and $1 \mathrm{~g}$ of sorbent. For higher Sephadex amounts, PACs were strongly retained and solvent volumes required to elute these components increased substantially. The influence of the flow rate on the SEC process was studied at 1 and $1.5 \mathrm{~mL} \mathrm{~min}^{-1}$. Higher values were not recommendable as generated significant deformations in the sorbent bed. In order to follow the evolution of PAC contents in the initial extracts, the washed solution and elution fractions taken every $2.5 \mathrm{~mL}$ were analyzed by the HPLC method. The process was more efficient at $1 \mathrm{~mL} \mathrm{~min}{ }^{-1}$ in terms of cleanup index as the major percentage of PAC was eluted within the first $2.5 \mathrm{~mL}$ fraction.

The overall performance of the Sephadex purification is evidenced in Fig. 2 which depicted a comparison of the UV chromatograms at $280 \mathrm{~nm}$ of (a) an initial cranberry extract and (b) the final eluted fraction. The separation program applied to this preliminary study was as follows: 0 to $25 \mathrm{~min}, \mathrm{~B}(\%) 10 \rightarrow 40$ (linear increase); 25 to $30 \mathrm{~min}, \mathrm{~B}(\%) 40 \rightarrow 90$ (lineal increase). It should be noted that this program was different to that finally selected as indicated in the experimental section. For raw 
cranberry extracts, B2, C1 and A2 were completely imbibed in the intense signals of other pholyphenolic components. Conversely, peaks of compounds such as A2 and B2 were clearly detected after purification ( $t_{R} 16.3$ and $6.6 \mathrm{~min}$, respectively). The repeatability of the whole manual SEC treatment was assessed with 5 independent replicates of homemade prepared cartridges. The RSD\% of overall areas of non-PAC and PAC fractions were 9 and $13 \%$, respectively.

From these preliminary manual assays it was concluded that the pass of solvents through the cartridge affected the homogeneity of the sorbent bed and, thus, the variability of results. An accurate control of flow rate and pressure was difficult so the cleanup repeatability was not entirely satisfactory. In order to improve the precision of the procedure and reuse the Sephadex sorbent further experiments were performed as explained in the experimental section. A volume of $1 \mathrm{~mL}$ of cranberry extract in methanol/water/HCl 60:39:1 (v:v:v) was loaded into the SEC column at a constant flow rate of $0.3 \mathrm{~mL} \mathrm{~min}{ }^{-1}$. The separation of monomeric- and oligomeric-rich fractions was carried out as follows: 0 to $30 \mathrm{~min}$, isocratic elution with methanol/water 30:70 (v:v) at $0.3 \mathrm{~mL} \mathrm{~min}^{-1} ; 30$ to $60 \mathrm{~min}$, isocratic elution with acetone/water $60: 40(v: v)$ at $0.3 \mathrm{~mL}$ $\mathrm{min}^{-1}$. The whole process was monitored at $280 \mathrm{~nm}$ and $520 \mathrm{~nm}$. Fig. 3 shows the SEC chromatogram of a fruit-based cranberry extract as an example. It was found that a first wide peak corresponded to the overall non-polymeric fraction after which the mobile phase changed into $70 \%$ acetone. Sephadex beads took ca. 20 min to shrink and allow the following elution of the fraction enriched with oligomeric species.

Fractions were collected into amber vials, then evaporated until dryness and redissolved in $1 \mathrm{~mL}$ extraction solution, filtered through Nylon $0.45 \mu \mathrm{m}$ filter and kept at $4^{\circ} \mathrm{C}$ until analysis by reversed-phase HPLC (see experimental section). Standard solutions of analytes were assayed by HPLC establishing the following retention times to be used for identification purposes: 15.8, 21.9, 19.1, 23.2, and 28.9 min for catechin, epicatechin, procyanidin $\mathrm{B} 2$, procyanidin $\mathrm{C} 1$, and procyanidin $\mathrm{A} 2$, respectively. Chromatograms of the initial (untreated) cranberry fruit extract, and methanol and acetone fractions are presented in Fig. 4. FLD signals of the initial extracts showed that the samples were rich in catechin and epicatechin, and procyanidin A2 was also detectable. Apart from flavanols, peaks of various hydroxybenzoic acids such as gallic, dihydroxybenzoic, vanillic, syringic or synaptic acids could also be recognized using the corresponding standards as a reference. The FLD chromatogram of the methanol fraction basically contained signals of side hydroxybenzoic acids, which were 
significantly smaller in size. The analysis of the acetone fraction, conveniently redissolved in the mobile phase, showed that the time range from 5 to $15 \mathrm{~min}$ was free of interfering phenolic acids, thus proving the performance of the cleanup method. Besides, peaks of flavanols, especially procyanidin A2, were maximized in comparison with the rest of components.

Apart from off-line coupling of SEC and reversed-phase processed, online coupling was also attempted. In this case, the PAC fraction containing the biologically active ingredients was directly introduced into the injection loop of the second HPLC system. Anyway, despite the great analytical possibilities of this approach, the high acetone concentration in the medium led to double peaks in the reversed-phase runs as a consequence of the lower polarity in comparison with the mobile phase. This issue may decrease the chromatographic performance so the off-line method seems to be more recommendable.

\section{Figures of merit}

Analytical parameters of the proposed method using fluorescence detection at $\lambda_{\text {exc }} 280$ and $\lambda_{\text {em }} 347 \mathrm{~nm}$ were established with standards of catechin, epicatechin, procyanidin $\mathrm{B} 2$, procyanidin $\mathrm{C}$, and procyanidin $\mathrm{A} 2$. Repeatabilities expressed as relative standard deviation (RSD\%) from 6 replicate injections of $10 \mathrm{mg} \mathrm{L}^{-1}$ standard solutions each compound were better than $1 \%$ and $0.4 \%$ in terms of retention time and peak area, respectively. Calibration curves were linear up to $100 \mathrm{mg} \mathrm{L}^{-1}$ (maximum assayed), with determination coefficients $\left(r^{2}\right)$ better that 0.999 . Limits of detection (LODs) at a signalto-noise ratio of 3 were below $0.15 \mathrm{mg} \mathrm{L}^{-1}$. Regarding the purification step, RSD\% of analyte peak areas in the extract fractions were better than $5 \%$. Exploratory studies of potential matrix effects from the comparison of sensitivities in pure synthetic and extracted matrix solutions suggested the absence of matrix deviations, with sensitivity ratios $(\%)$ of ca. $100 \%$. As a conclusion, figures of merit of the proposed method were fully compatible with typical levels of analytes found in fruit extracts and nutraceutical samples.

\section{Determination of proanthocyanins in cranberry samples}

The method developed here was applied to the determination of proanthocyanins in cranberry samples. Chromatograms were acquired at $\lambda$ ex $280 \mathrm{~nm} / \lambda$ em $347 \mathrm{~nm}$ by 
FLD spectroscopy to enhance the selectivity and sensitivity of the detection. Three independent extractions of each sample were carried out as described in the experimental section using $10 \mathrm{~mL}$ of methanol/water/hydrochloric acid (60:39:1 (v:v:v)) solution.

As an example, quantitative results have been summarized in Fig. 5. The sample description can be found in the experimental section. Error bars indicated the standard deviation of results. It can be seen that a group of samples (e.g. C02, C08, C13, E1, etc.) showed a pattern in which A2 was the most abundant component, in some cases with concentrations higher that $5 \mathrm{mg} \mathrm{g}^{-1}$. They also contained high concentrations of B2 and epicatechin. Another type of samples such as C09, C10 and E2 were rich in monomers, especially catechin with concentrations of $10 \mathrm{mg} \mathrm{g}^{-1}$ or higher. A third pattern corresponded to samples such as $\mathrm{C} 11$ and $\mathrm{C} 12$, in which $\mathrm{A} 2$ was negligible but B2 and monomers were detected at concentrations higher than $7 \mathrm{mg} \mathrm{g}^{-1}$.

\section{Conclusions}

Size-exclusion chromatography (SEC) coupled to reversed-phase liquid chromatography with UV absorption and fluorescence detection (HPLC-UV-FLD) was here successfully used for the determination of flavanols in cranberry extract samples. The proposed method did not require complex and expensive instrumental platforms such as LC-MS but exploited typical equipment available in most of the analytical laboratories. Besides, the reusable nature of the SEC step implemented in a packed column was highly advantageous from both practical and economical points of view. The performance of Sephadex LH-20 as the sorbent to fraction simple phenolic and oligomeric components was thoroughly assessed on solid-phase extraction and SEC formats. Limitations dealing the poor repeatability of manual homemade cartridges could be overcome in the SEC approach based on a reusable Sephadex column. As a result, fractions rich in monomeric and oligomeric components were efficiently separated based on sieving process in which changes in the sieving size were induced from changes in the solvent polarity. The method developed here for the determination of flavanols in cranberry-based samples can easily be adapted to other fruit matrices as a complementary strategy to facilitate their characterization, classification and authentication. Further work for online coupling of size-exclusion and reversed-phase separation as a $2 \mathrm{D}$ chromatography will offer new opportunities in the study of complex food matrices. 


\section{Acknowledgement}

The authors gratefully acknowledge the financial support received from Spanish Ministry of Economy and Competitiveness under the projects CTQ2014-65324 and CTQ2015-63968-C2-1-P, and from the Agency for Administration of University and Research Grants (Generalitat de Catalunya, Spain) under the projects 2014 SGR-377 and 2014 SGR-539.

\section{Compliance with Ethical Standards}

Conflict of Interest: The first author Inal Bakhytkyzy, the second author Oscar Nuñez and the corresponding author Javier Saurina declare that they have no conflict of interest.

Ethical Approval: This article does not contain any studies with humans and animals performed by any of the authors.

Informed Consent: Not applicable.

\section{References}

Bakhytkyzy I, Nunez O, Saurina J (2018) Determination of flavanols by liquid chromatography with fluorescence detection. Application to the characterization of cranberry-based pharmaceuticals through profiling and fingerprinting approaches. J Pharm Biomed Anal 156:206

Bindon KA, Carew AL, Mierczynska-Vasilev A, Kassara S, Kerslake F, Smith PA (2016) Characterization of macromolecular complexes in red wine: Composition, molecular mass distribution and particle size. Food Chem 199:838

Carrasco-Pancorbo A, Gomez-Caravaca AM, Segura-Carretero A, Cerretani L, Bendini A, Fernandez-Gutierrez A (2009) Use of capillary electrophoresis with UV detection to compare the phenolic profiles of extra-virgin olive oils belonging to Spanish and Italian PDOs and their relation to sensorial properties. J Sci Food Agric 89:2144 
Coppo E, Marchese A. (2014) Antibacterial Activity of Polyphenols. Curr Pharm Biotechnol 15:380-390.

Cote J, Caillet S, Doyon G, Sylvain JF, Lacroix M (2010) Analyzing cranberry bioactive compounds. Crit Rev Food Sci Nutr 50:872

Guay DRP (2009) Cranberry and Urinary Tract Infections. Drugs 69:775-807

Diaz-Garcia MC, Obon JM, Castellar MR, Collado J, Alacid M (2013) Quantification by UHPLC of total individual polyphenols in fruit juices. Food Chem 138:938

Duval A, Averous L (2016) Characterization and Physicochemical Properties of Condensed Tannins from Acacia catechu. J Agric Food Chem 64:1751

van Dooren I, Foubert K, Theunis M, Naessens T, Pieters L, Apers S (2018) Advantages of a validated UPLC-MS/MS standard addition method for the quantification of A-type dimeric and trimeric proanthocyanidins in cranberry extracts in comparison with well-known quantification methods. J Pharm Biomed Anal 148:32

Gomez-Plaza E, Olmos O, Bautista-Ortin AB (2016) Tannin profile of different Monastrell wines and its relation to projected market prices. Food Chem 204:506

He X, Rui HL (2006) Cranberry phytochemicals: isolation, structure elucidation, and their antiproliferative and antioxidant activities. J Agric Food Chem 54:70697074

Howell A (2007) Bioactive compounds in cranberries and their role in prevention of urinary tract infections. Mol Nutr Food Res 51:732-737

Krenek K, Marhol P, Peikerova Z, Kren V, Biedermann D (2014) Preparatory separation of the silymarin flavonolignans by Sephadex LH-20 gel. Food Res Int $65: 115$

Koerner JL, Hsu VL, Lee JM, Kennedy JA (2009) Determination of proanthocyanidin A2 content in phenolic polymer isolates by reversed-phase high-performance liquid chromatography. J Chromatogr A 1216:1403 
Kraemer-Schafhalter A, Fuchs H, Pfannhauser W (1998) Solid-phase extraction (SPE) a comparison of 16 materials for the purification of anthocyanins from Aronia melanocarpa var nero. J Sci Food Agric 78:435

Lee J (2013) Proanthocyanidin A2 purification and quantification of American cranberry (Vaccinium macrocarpon Ait.) products. J Funct Foods 5:144

Lerma-Herrera MA, Nunez-Gastelum JA, Ascacio-Valdes J, Aguilar CN, RodrigoGarcia J, Diaz-Sanchez AG, Alvarez-Parrilla E, de la Rosa LA (2017)

Estimation of the Mean Degree of Polymerization of Condensed Tannins from the Kernel and Shell of Carya illinoinensis by HPLC/MS and Spectrophotometric Methods. Food Anal Meth 10:3023

Li Q, Wang XY, Chen J. Liu CM, Li T, McClements DJ, Dai TT, Liu JY (2016) Antioxidant activity of proanthocyanidins-rich fractions from Choerospondias axillaris peels using a combination of chemical-based methods and cellularbased assay. Food Chem 208:309

Li ZL, Shi WX, Cheng LD, Pan, SH, Wang, CJ (2018) Screening of the phenolic profile and their antioxidative activities of methanol extracts of Myrica rubra fruits, leaves and bark. J Food Meas Charact 12:128

Lin LZ, Sun JH, Chen P, Monagas MJ, Harnly JM (2014) UHPLC-PDA-ESUHRMSn Profiling Method To Identify and Quantify Oligomeric Proanthocyanidins in Plant Products. J Agric Food Chem 62:9387

Monagas M, Quintanilla-Lopez JE, Gomez-Cordoves C, Bartolome, B, Lebron-Aguilar, R (2010) MALDI-TOF MS analysis of plant proanthocyanidins. J Pharm Biomed Anal 51:358

Navarro M, Nunez O, Saurina J, Hernandez-Cassou S, Puignou L (2014) Characterization of Fruit Products by Capillary Zone Electrophoresis and Liquid Chromatography Using the Compositional Profiles of Polyphenols: Application to Authentication of Natural Extracts. J Agric Food Chem 62:1038

Orejola J, Matsuo Y, Saito Y, Tanaka T (2017) Characterization of Proanthocyanidin Oligomers of Ephedra sinica. Molecules 22:1308 
Pappas E, Schaich KM (2009) Phytochemicals of Cranberries and Cranberry Products: Characterization, Potential Health Effects, and Processing Stability. Crit Rev Food Sci Nutr 49:741-781

Parveen S, Memon SQ, Siyal AN, Memon N, Khuhawar MY (2016) Large-Volume Sample Staking of Rice Polyphenols Prior to Their Determination by Nonaqueous Capillary Electrophoresis. Food Anal Meth 9:2152

Rue EA, Rush MD, van Breemen RB (2018) Procyanidins: a comprehensive review encompassing structure elucidation via mass spectrometry. Phytochem Rev 17:1

Sawalha SMS, Arraez-Roman D, Segura-Carretero A, Fernandez-Gutierrez A (2009) Quantification of main phenolic compounds in sweet and bitter orange peel using CE-MS/MS. Food Chem 116:567

Verardo V, Cevoli C, Pasini F, Gomez-Caravaca AM, Marconi E, Fabbri A, Caboni MF (2015) Analysis of Oligomer Proanthocyanidins in Different Barley Genotypes Using High-Performance Liquid Chromatography-Fluorescence Detection-Mass Spectrometry and Near-Infrared Methodologies. J Agric Food Chem 63:4130

Wang CJ, Zhao JB, Chen F, Cheng YQ, Guo AH (2012a) Separation, Identification, and Quantitation of Phenolic Acids in Chinese Waxberry (Myrica Rubra) Juice by HPLC-PDA-ESI-MS. J Food Sci 77:C272

Wang CJ, Zuo YG (2011) Ultrasound-assisted hydrolysis and gas chromatography-mass spectrometric determination of phenolic compounds in cranberry products. Food Chem 128: 562

Wang CJ, Zuo YG, Vinson JA, DengYW (2012b) Absorption and excretion of cranberry-derived phenolics in humans. Food Chem 132:1420

White BL, Howard LR, Prior RL (2010) Proximate and Polyphenolic Characterization of Cranberry Pomace. J Agric Food Chem 58:4030

www.sigmaaldrich.com/content/dam/sigma-aldrich/docs/SigmaAldrich/General_Information/1/ge-size-exclusion-chromatography.pdf. Chapter 6 Sephadex LH-20: size exclusion chromatography in organic solvents pp 83-88 (accessed 6/26/2018) 


\section{Figure captions}

Figure 1. Surface responses for the evaluation of the influence of acidity and methanol percentage on the flavanol extraction. (a) procyanidin A2; (b) epicatechin; (c) procyanidin B2; (d) overall flavanol extraction.

Figure 2. Chromatograms at $280 \mathrm{~nm}$ corresponding to (a) an initial (untreated) cranberry extract and (b) the final eluted fraction from the Sephadex cartridge.

Figure 3. Size exclusion chromatogram at $280 \mathrm{~nm}$ of cranberry fruit extract eluted with $30 \%$ methanol/water 30/70, (v:v) and acetone/water 70/30 (v:v). $1=$ monomeric fraction, 2 = polymeric fraction.

Figure 4. Study of the performance of the SEC procedure for sample treatment. Chromatograms by FLD at $\lambda$ ex $280 \mathrm{~nm} / \lambda$ em $347 \mathrm{~nm}$ corresponding to (a) extract of cranberry fruit, (b) fraction of monomers eluted with methanol/water 30:70 (v:v), (c) fraction of oligomers eluted with acetone/water 70:30 (v:v).

Figure 5. Determination of the composition of cranberry samples. Analyte assignation: $1=$ catechin, $2=$ epicatechin, $3=$ procyanidin $\mathrm{B} 2,4=$ procyanidin $\mathrm{A} 2$. 
(a)
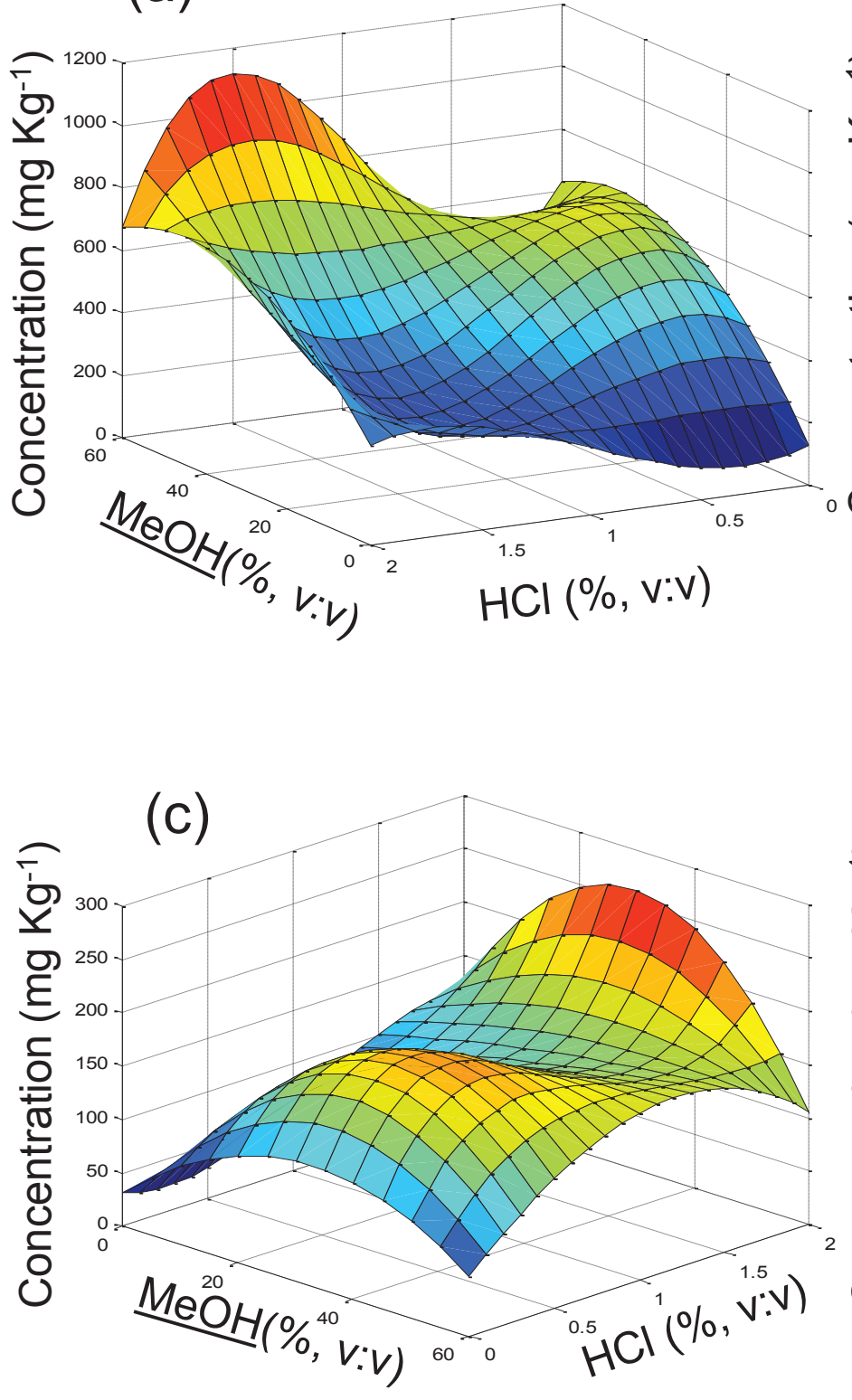

(b)
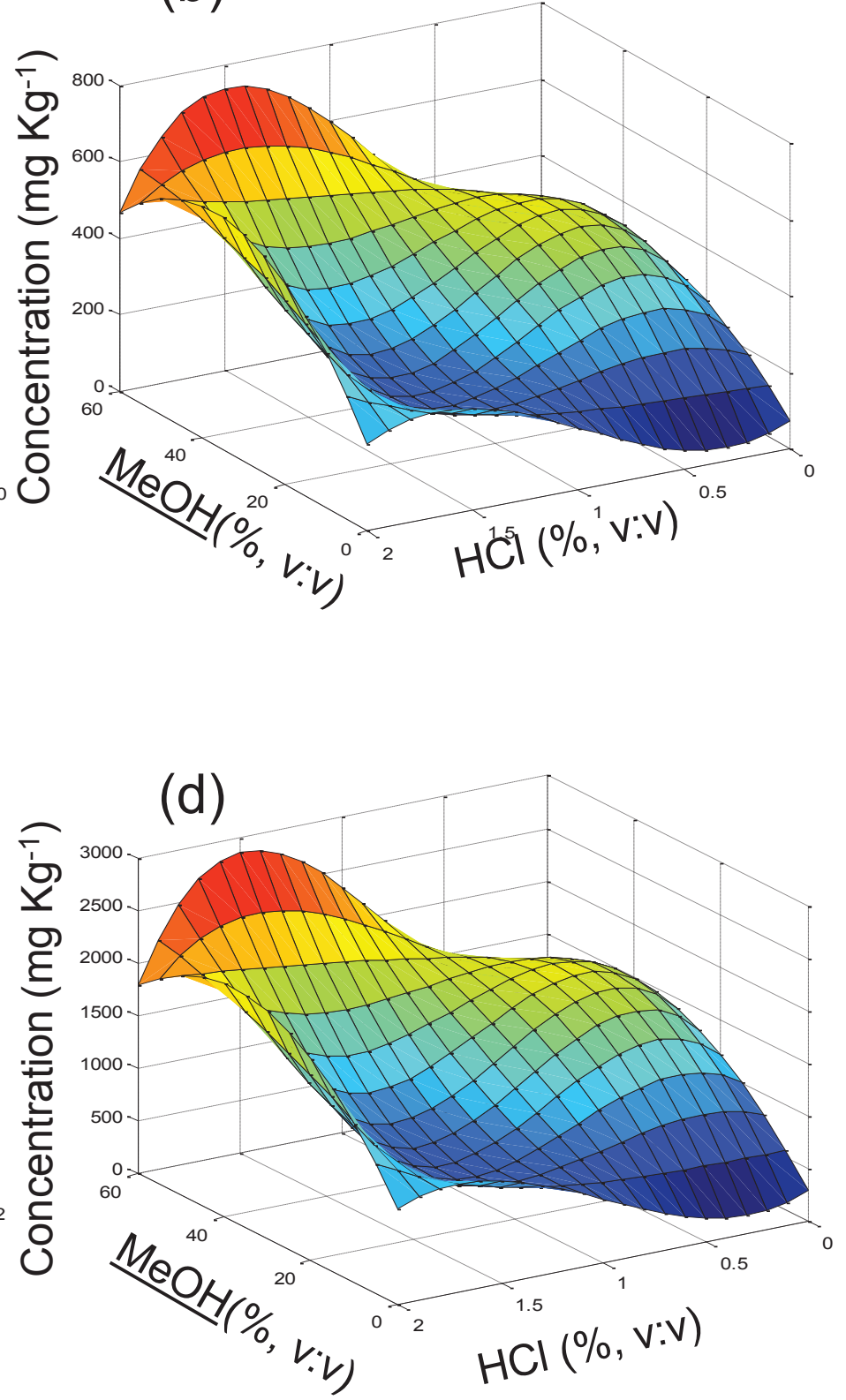


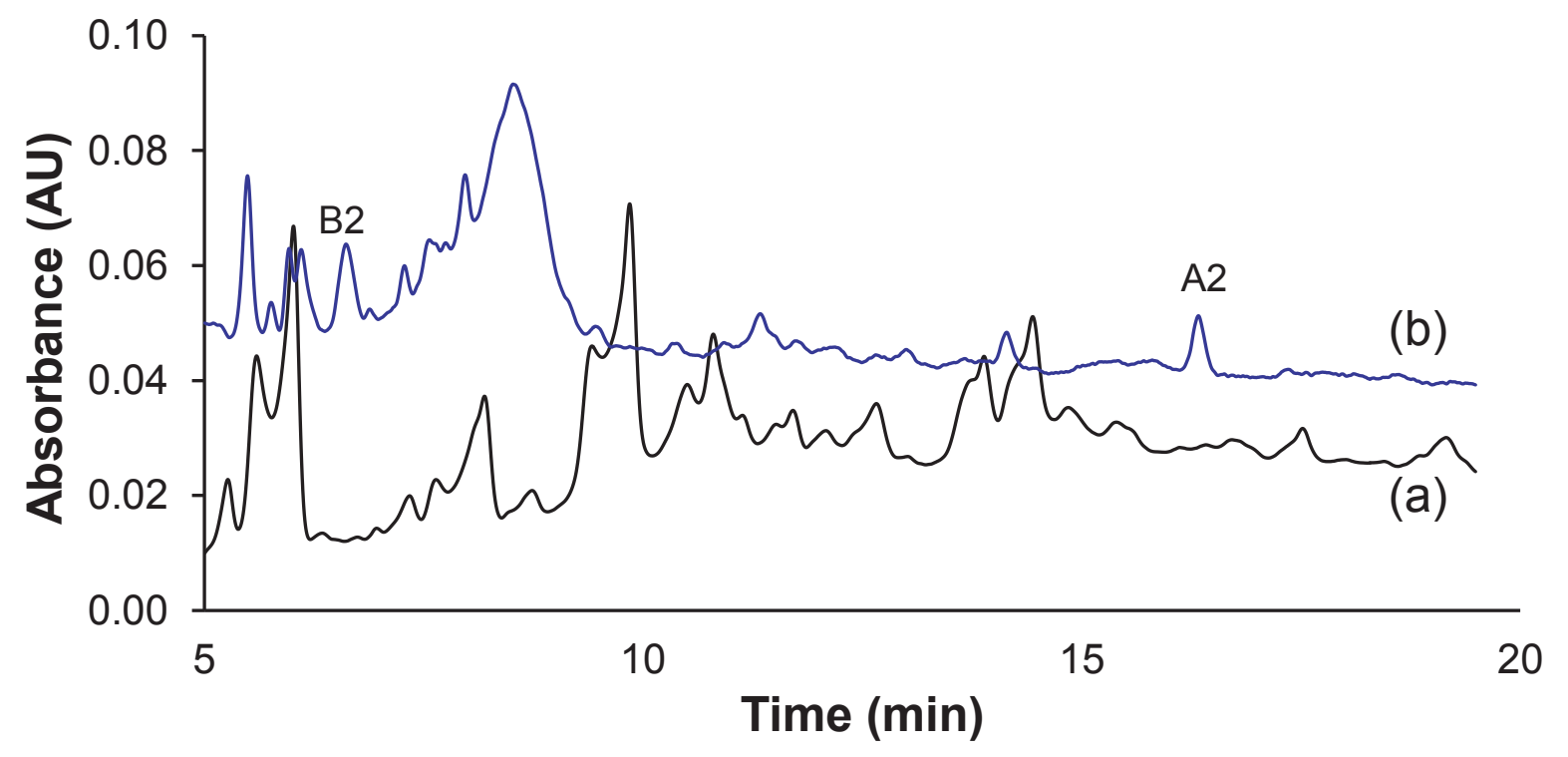




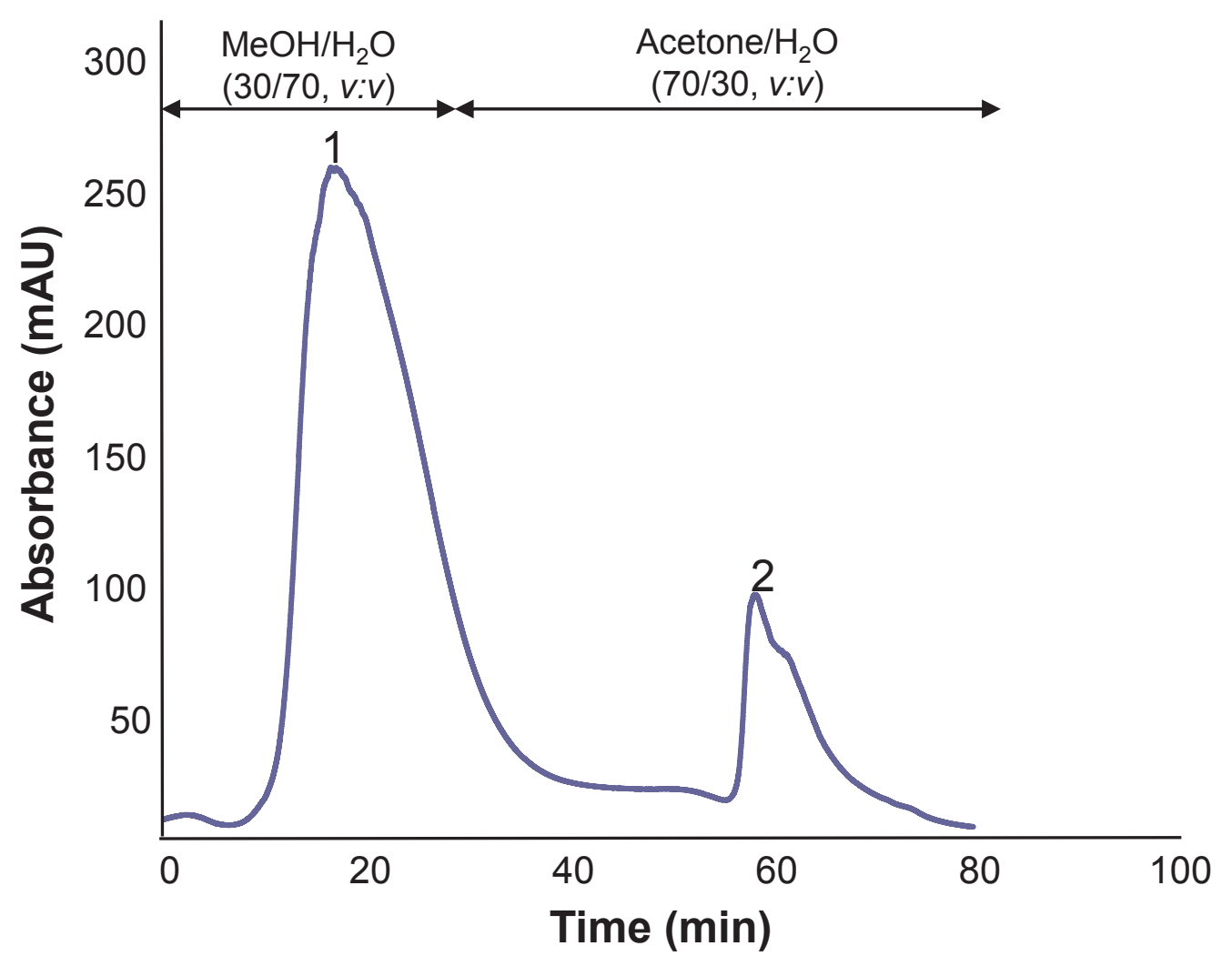




\section{Fluorescence (Arbitrary)}

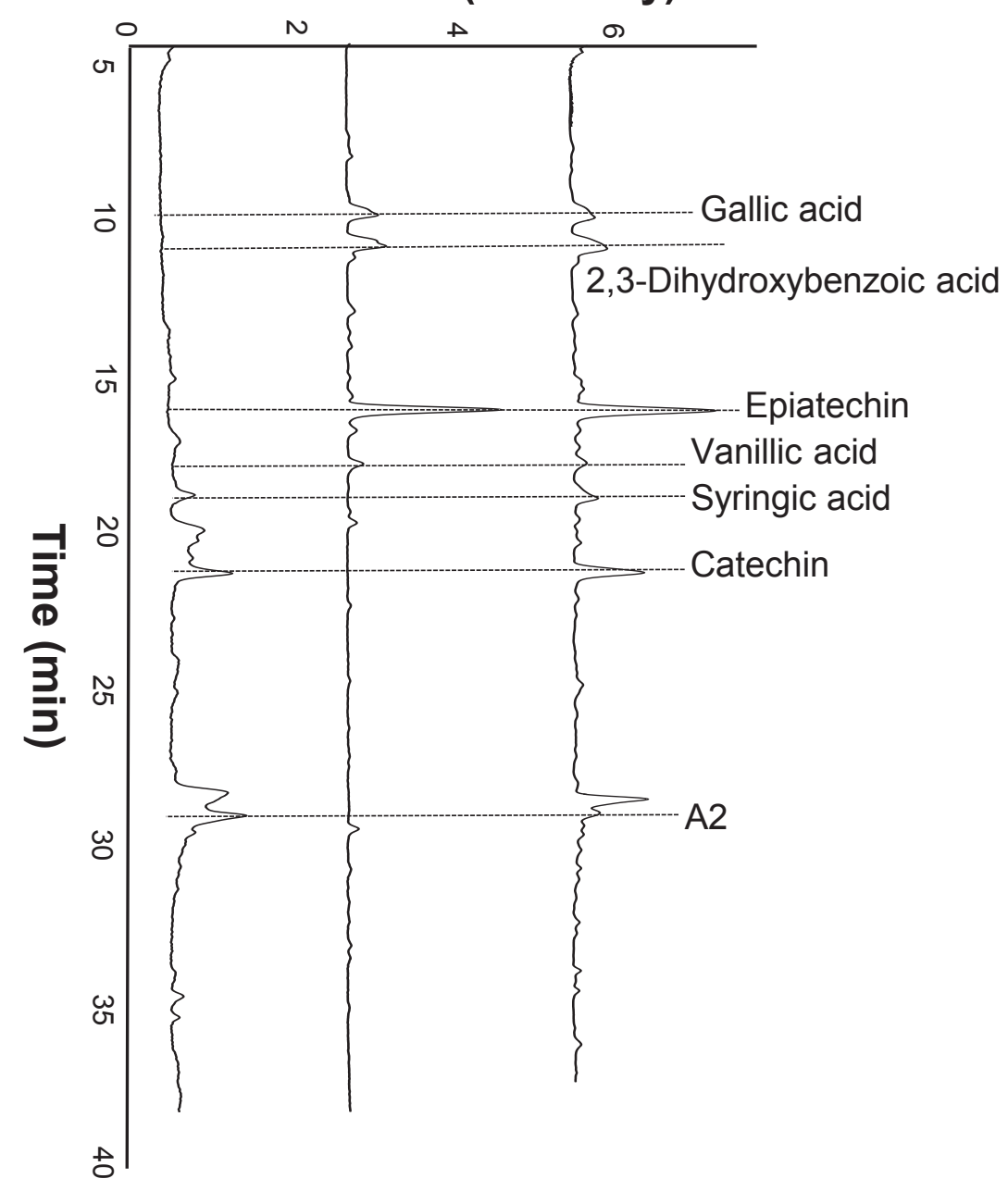




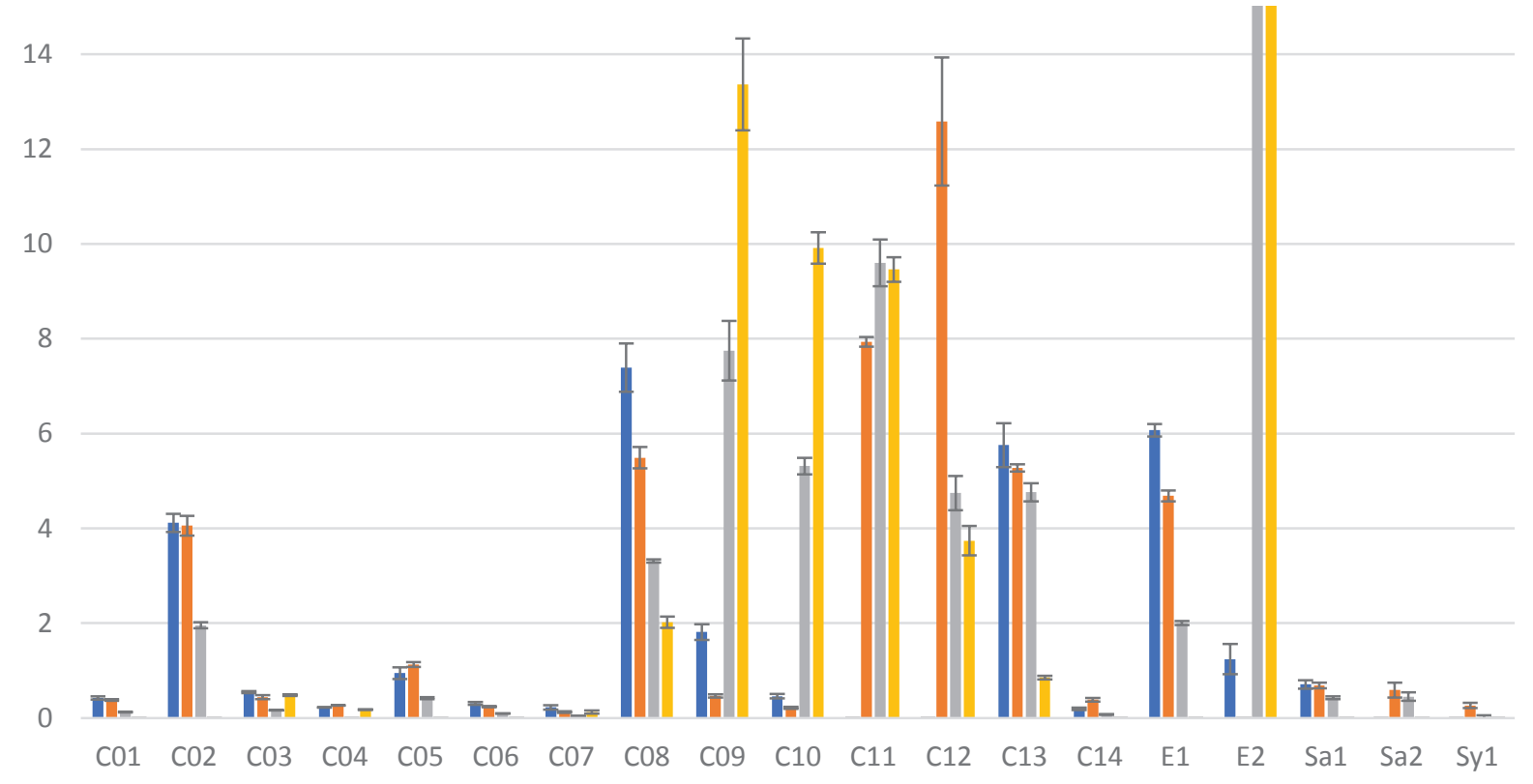

